machine using reagents from Hema (Russia). Statistical processing of the data was performed on a personal computer using the software package 'Statistica 6'.

Results and discussion Determining plasma concentration of endothelin helps to establish the diagnosis of heart failure in the early stages of the disease. The study of endothelin content depending on the form of cardiomyopathy revealed that the average content of endothelin was unreliable higher in children with hypertrophic cardiomyopathy and corresponded to the upper limit of normal $(1.0 \pm 0.2 \mathrm{mmol} / \mathrm{l}$, $\mathrm{p}>0.05)$, compared with the rate of children with dilated cardiomyopathy $(0,86 \pm 0.1 \mathrm{mmol} / \mathrm{l}, \mathrm{p}>0.05)$. However, according to the results of our studies, an increase in the level of endothelin as the progression of the disease was found. Thus, in patients with a disease duration of 5 years, the level of endothelin was $1.3 \mathrm{mmol} / \mathrm{l}$, in patients with a disease duration of 9 years, this figure was $1.7 \mathrm{mmol} / \mathrm{l}$, and in a patient with a disease duration of 15 years - 1.98 $\mathrm{mmol} / \mathrm{l}$, which is 2 times the norm. A correlation analysis between the prescription of CMP, the severity of chronic heart failure and the level of endothelin revealed a strong direct correlation $(\mathrm{r}=+0.97)$, which indicates the predictive value of endothelin. From this it follows that determining the plasma concentration of endothelin helps to establish the diagnosis heart failure in the early stages of the disease.

Conclusion As the disease progresses, an increase in the level of endothelin is a reliable prognostic criterion for the severity of chronic heart failure in children with cardiomyopathy.

\section{P58 THE IMPORTANCE OF MEDICAL AND BIOLOGICAL FACTORS IN THE DEVELOPMENT OF CARDIOMYOPATHY IN CHILDREN}

${ }^{1}$ Nilufar Akhmedova*, 2,1Dilorom Akhmedova, ${ }^{2}$ Flora Inoyatova. 'Tashkent Pediatric Medical Institute, Tashkent, Uzbekistan; ${ }^{2}$ Republican Specialized Scientific and Practical Medical Center of Pediatrics, Tashkent, Uzbekistan

10.1136/archdischild-2019-epa.413

The health and well-being of people today are influenced by many factors that are determining. Studies show that the main risk factors for cardiovascular diseases, often occur in childhood, are relatively stable, since their presence is confirmed by repeated studies conducted in an adult state.

The purpose To evaluate the role of medical-biological factors in the development of cardiomyopathy in children

Materials and methods The study involved 86 patients with diagnoses of hypertrophic, dilatation and restrictive cardiomyopathy, who were hospitalized in the cardio-rheumatology department of the Republican Specialized Scientific and Practical Medical Center of Pediatrics, the Republican Children's Multipurpose Medical Center of the Republic of Karakalpastan. The diagnosis was made on the basis of complaints, anamnesis data (obstetric history of the mother, history of the child's life and illness, hereditary predisposition, past diseases, nature of the course and duration of the disease), clinical and functional (ECG, EchoCG), laboratory examination methods.

Results The study of biomedical factors contributing to the development of cardiomyopathy in children has shown that the disease is sex-related and is more common in boys (60\%). In $10 \%$ of patients with CMP, the age of mothers was over
35 years old at the time of birth of this child. Nearly related marriages were registered in $25 \%$ of cases, hereditary burden in $7.5 \%$ of patients. Studies have shown that one determinant that has a significant impact on the development of the ILC is also the burden of obstetric history of mothers. Thus, the study of obstetric history of mothers indicates the birth of premature babies, the presence of stillbirths, miscarriages in early pregnancy. This pregnancy proceeded against the background of anemia and pre-eclampsia of the first or second half of pregnancy. Most of the mothers suffered from extragenital diseases, among which were more frequent kidney and heart diseases (chronic pyelonephritis and carditis), allergic reactions. The number of mothers who had ARI during pregnancy was $85 \%$. The debut of the disease was associated with sudden cardiac arrest in $3.3 \%$ of the child; syncopal state during physical exertion in $6.67 \%$ of children and with postponed carditis in $13.3 \%$ of children.

Thus, among the biomedical factors that influence the development of cardiomyopathy the most closely related marriage, hereditary predisposition, obstetric history and unfavorable pregnancy, the presence of extragenital diseases in the mother, the age of the mother over 35, and childhood diseases of viral etiology are most significant.

\section{P59 RELATIONSHIP BETWEEN ORAL HEALTH AND HYPERTENSION IN ADOLESCENTS}

Larisa Kolesnikova, Anna Pogodina, Olga Berdina, Olga Valyavskaya, Lilia Zurbanova, Lyubov Rychkova*. Scientific Centre for Family Health and Human Reproduction Problems, Irkutsk, Russian Federation

10.1136/archdischild-2019-epa.414

Aim to characterize oral health in adolescents with hypertension and evaluate presence of significant associations between hypertension and main dental diseases.

Methods A case-control study was conducted. A total of 130 subjects aged 10-17 were included in the study. The group of 'cases' included 65 adolescents with hypertension, verified by ABPM, and control group included the same number of adolescents with normal blood pressure levels, compatible by the sex and age. All adolescents underwent anthropometric measurements with BMI calculation, dental examination (caries intensity index, gingivitis index, complex dental plaque index, oral hygiene index). Binary logistic regression, adjusted for BMI z-scores and oral hygiene indexes, was used to assess the relationship between hypertension and presence and severity of oral diseases in adolescents.

Results Frequency of dental caries and periodontitis was higher in the group of adolescents with hypertension in comparison with the controls $(76.9 \%$ vs. $49.2 \%$ for caries, and $40 \%$ vs. $9.2 \%$ for periodontitis, $\mathrm{p}=0.001$ for both). Signs of moderate to severe gingivitis were noticed in $18.5 \%$ adolescents with hypertension and only in $3.1 \%$ adolescents from the control group $(p=0.009)$. Poor oral hygiene was detected in $43.1 \%$ adolescents with hypertension and in $21.5 \%$ adolescents from the control group $(p=0.002)$. Presence of hypertension was significantly associated with caries and periodontitis, but not with gingivitis in adjusted regression models (OR 3 [1.3 7.1], $\mathrm{p}=0.012$ for caries, and OR 3.9 [1.4 - 10.5], $\mathrm{p}=0.007$ for periodontitis). Moreover, hypertension was associated with more severe damage of periodontium (OR 3 [1.6 - 5.6], $\mathrm{p}=0.001)$. 
Conclusion Results of this study showed, that hypertensive adolescents have poorer oral health than adolescents with normal BP level. This substantiates the necessity of multidisciplinary approach to management of this group of patients with participation of both pediatric physicians and dentists.

\section{P60 CLINICAL CHARACTERISTICS, COMPLICATIONS, AND ANTICOAGULATION IN CHILDREN WITH INFECTIVE ENDOCARDITIS: A SINGLE CENTER EXPERIENCE FROM TURKEY}

${ }^{1}$ Pelin Kosger, ${ }^{2}$ Tugcem Keskin, ${ }^{2}$ Hikmet Kiztanir, ${ }^{3}$ Birgul Kirel, ${ }^{2}$ Birsen Ucar*. ${ }^{*}$ Eskisehir Osmangazi Universty, Faculty of Medicine, department of Pediatric Cardiology, Eskisehir, Turkey; ${ }^{2}$ Eskisehir Osmangazi Universty, Faculty of Medicine, department of Pediatric Cardiology, Eskisehir, Turkey; ${ }^{3}$ Eskisehir Osmangazi Universty, Faculty of Medicine, department of Pediatric Endocrinology, Eskisehir, Turkey

10.1136/archdischild-2019-epa.415

Objective In this study, the data of our patients diagnosed with infective endocarditis (IE) between 2010-2018 at the Eskişehir Osmangazi University Faculty of Medicine Hospital, Department of Pediatric Cardiology were retrospectively obtained.

Methods Demographic data, the presence of a predisposing cardiac disease, presentation with complaints, clinical and laboratory findings, blood culture results, treatment plans, evolving complications, and the echocardiographic data of eleven patients were examined. The relevant features of the patients with complications, of those having anticoagulant therapy in addition to antibiotherapy, those who underwent surgery, and those who showed a mortality course were determined.

Results The youngest patient was 7 months old and the oldest was 14 years old $(7.5 \pm 4.6$ years $)$. All of the cases had congenital heart anomalies: four had cyanotic congenital heart disease (CHD), four had a ventricular septal defect (VSD), two had a bicuspid aortic valve (BAV), and one patient had a secundum atrial septal defect (ASD). There were no cases with rheumatic heart disease. Three patients with cyanotic CHD were treated with corrective cardiac surgery. A total of eight patients had embolic findings; five at the time of admission and three at follow-up. Nine patients received antibiotics prior to admission. Echocardiography showed vegetation in nine patients. Anticoagulant treatment was applied to 2 patients with mitral valve and aortic valve vegetation in addition to antibiotic treatment. The most common microorganism in the blood culture was coagulase negative staphylococcus with 5 cases and 2 of them were identified as staph. epidermidis. The second most common microorganism was the viridans group streptococcus with 4 cases. Four patients underwent early surgical treatment, one patient died due to multiple organ failure caused by systemic embolization, and one patient died due to sudden hemodynamic instability in the first week of follow-up.

Conclusion IE is a serious disease with life-threatening complications, where the number of at-risk patients due to $\mathrm{CHD}$ is gradually increasing and thus the mortality rate has not decreased despite improved medical and surgical treatment procedures. Early initiation of appropriate antibiotic treatment following early diagnosis is has great importance in preventing mortality.Considering the complications that may be caused by early surgical intervention, anticoagulant therapy, which is combined with antibiotherapy in appropriate patients, can be tried as a useful option prior to committing to early surgery.

\section{P61 ATRIOVENTRICULAR BLOCK IN CHILDREN - SOMETIME IS A CHALLENGE}

${ }^{1,2}$ Gabriela Doros, ${ }^{1,2}$ Andrada Mara Ardelean* ${ }^{1,2}$ Anca Popoiu, ${ }^{1,2}$ Cristina Olariu ${ }^{1,2}$ Ramona Stroescu, 1,2 Mihai Gafencu. 'Victor Babes University of Medicine and Pharmacy, Timisoara, Romania; ${ }^{2}$ IIIrd Pediatric Clinic Emergency Hospital for Children Louis Turcanu, Timisoara, Romania

\subsection{6/archdischild-2019-epa.416}

Introduction Atrioventricular(AV) blocks in children may become a challenge in some situations and the aim is to present three different cases admitted in our Department.

Material and method A 12 yo girl was admitted for precordial pain during a respiratory infection. An 8 yo boy was admitted for a syncope in the time he was laying in bed near his father. The third case was a 14 yo girl, known from the age of 2,6 yo with gr. III AV block, implanted with an abdominal pace maker(PM) with epicardial leads, changed in 2016 with a bicameral PM, programmed DDD with $60 \mathrm{~b} / \mathrm{min}$; she was admitted for intense fatigue. All of them performed cardiac and arrhythmology examination.

Results The 12 yo girl presented on ECG, grade I AV block, with a PQ interval of $0.30 \mathrm{sec}$. Holter ECG revealed gr I AV block with different PQ intervals, the maximum length being 0.37 sec. and paroxysmal Gr II Mobitz I Block during night. Being asymptomatic, the decision was just to be followed at 6 mo. The boy with the syncope presented on Holter ECG, Gr II Mobitz I AV block during night, that may be a normal condition in asymptomatic children. Both patients had structurally normal heart and function. The third patient presented tachycardia $122 \mathrm{~b} / \mathrm{min}$ and weakness. She had cardiac dissynchrony, EF 53\%, gr I mitral regurgitation, ASD and reduced GLS. The PM was changed to VVI with $50 \mathrm{~b} / \mathrm{min}$. She felt better, but dizzy. Atrial conduction was 120/min and Ventricular spike were at $50 / \mathrm{min}$. Next day PM was upgraded to VVIR, with atrial conduction at $120 / \mathrm{min}$ and ventricular activity at $73 \mathrm{~b} /$ min. Ecogocardiography, EF improved and dissyncrony disappeared. The patient felt much better, being able to be discharged.

Conclussions Gr I AV block, when prolonged PQ interval more than $0.30 \mathrm{sec}$, needs to be investigated. Asymptomatic paroxistic gr II Mobits I block during sleep, has to be monitored. The new concept of PM Syndrome has to be checked and fixed to be safe for the patient. Simple cases in different situations become a challenge for the pediatric cardiology doctor.

\section{P62 A REVIEW OF NEAR INFRA-RED SPECTROSCOPY}

Peter Donnelly*, Miriam Fine-Goulden. Evelina London Children's Hospital, London, UK

10.1136/archdischild-2019-epa.417

Near infra-red spectroscopy (NIRS) is an exciting non-invasive, portable, continuous modality used to assess regional tissue oxygen saturations, expressed as a percentage. The use of NIRS in clinical practice is relatively recent, with its role in measuring cerebral oxygenation originally described in the 1970s.

The primary application of NIRS in paediatrics is in monitoring for signs of reduced or differential tissue perfusion, particularly post cardiac surgery. 